\title{
Toxicidad del misoprostol sobre la gestación. Revisión de la literatura
}

\author{
MARÍA FERNANDA CAVIERES ${ }^{\mathrm{a}}$
}

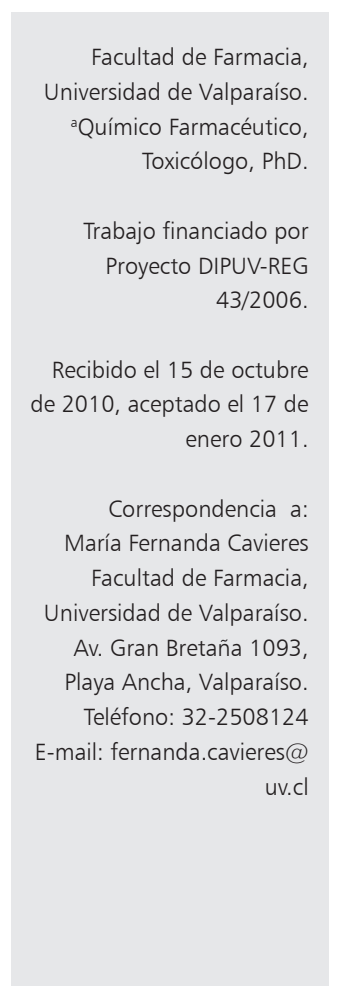

\section{Developmental toxicity of misoprostol. An update}

Misoprostol, a synthetic analog of prostaglandin $E_{1}$, is currently used in Chile and other countries as an antiulcer medication, mainly for the prevention of non-steroidal anti-inflammatory-induced gastric ulcers. Due to its uterotonic properties, it is also indicated in obstetrics for induction of labor and termination of pregnancy. In this last case, misoprostol is either used alone or in combination with other oxytocic drugs such as methotrexate or mifepristone. The use of misoprostol as an abortifacient agent is considered to be safe since it rarely causes serious side effects. However up to $15 \%$ of misoprostol-induced-abortions may not be successful, even under medical supervision, leading to in utero exposure to the drug and to the induction of a series of birth defects including limb and joints defects and Moebius syndrome. Reports from the nineties failed to show a strong epidemiological association between in utero drug exposure and induction of defects, a situation that has changed now that the number of cases reported has increased. Since the practice of abortion is illegal in Chile, many women turn to off-medical procedures to interrupt their pregnancy and use misoprostol as an easy and cheap alternative, readily available in the INTERNET. The lack of medical supervision in these cases may lead to situations that favor the induction of congenital defects. Here, we present an updated review of scientific data, to evaluate the risk of birth defects in babies exposed to the drug during pregnancy termination failed attempts.

(Rev Med Chile 2011; 139: 516-523).

Key words: Abnormalities, drug induced; Abortion, induced; Misoprostol.

$\mathrm{M}$ isoprostol, un análogo sintético de la prostaglandina $\mathrm{E}_{1}$ (Figura 1), es utilizado en Chile como medicamento antiulceroso, para prevenir úlceras gástricas inducidas por anti-inflamatorios no esteroidales. En otros países, tiene también indicación gineco-obstétrica ya que su actividad uterotónica lo hace eficaz como inductor de parto y como agente abortivo. En este último caso, el misoprostol se utiliza especialmente en el primer trimestre de embarazo, solo o asociado a otros oxitócicos como metotrexato o mifepristona ${ }^{1}$.

El uso de misoprostol como agente abortivo es considerado seguro dado que raramente causa efectos laterales serios o prolongados ${ }^{1}$. Sin embargo, aproximadamente $15 \%$ de los abortos inducidos por misoprostol pueden fallar, llevando a exposición del feto a la droga. La Food and Drug Administration (FDA) de los Estados Unidos de Norteamérica clasifica al medicamento en la categoría $\mathrm{X}$, según el riesgo de inducción de toxicidad sobre la gestación, dado que éste puede causar defectos congénitos que incluyen artrogriposis y otros defectos de extremidades, anormalidades cerebrales, gastrosquisis y síndrome de Moebius ${ }^{2}$. Sin embargo, algunos de los primeros reportes en la literatura mostraron una pobre asociación entre la exposición in utero al medicamento y la inducción de defectos del desarrollo ${ }^{3,4}$. Asimismo, un informe de una organización no gubernamental 


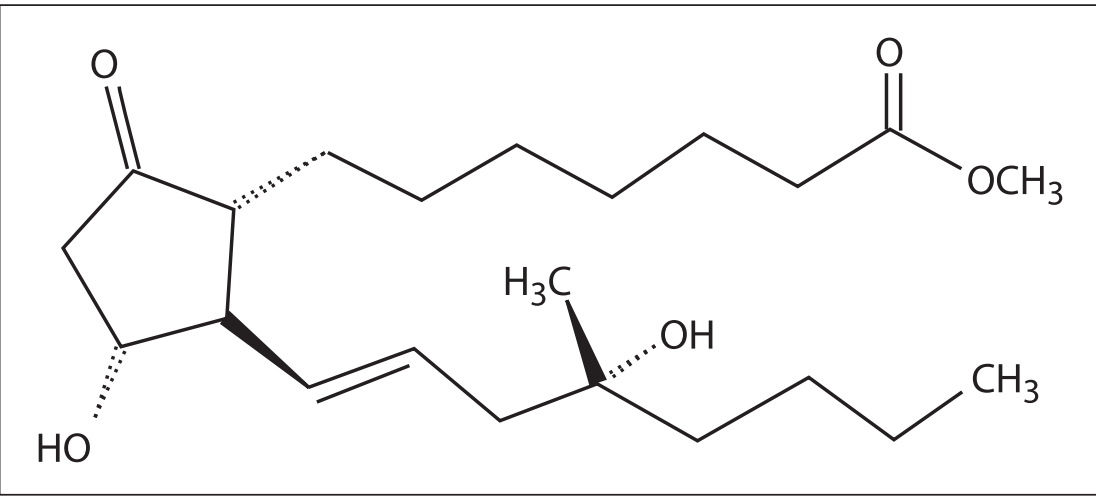

Figura 1. Estructura química del misoprostol. de Estados Unidos de Norteamérica, en el que se revisa la evidencia que liga la exposición a misoprostol con teratogenicidad, concluye que aunque el riesgo relativo de malformaciones parece real, el riesgo absoluto es bajo ${ }^{5}$.

Frente a esta aparente controversia, en esta revisión se presenta una actualización sobre la toxicidad que ejerce misoprostol durante la gestación con el fin de dar a conocer el riesgo de inducción de defectos del desarrollo en niños expuestos al medicamento durante intentos fallidos de abortos.

\section{Propiedades físicoquímicas}

El misoprostol (metil ester del ácido $(11 \alpha, 13 \mathrm{E})$ 11,16-dihidroxi-16-metil-9-oxoprost-13-en-1oico) es un análogo sintético de la prostaglandina $\mathrm{E}_{1}$. Es un racemato de cuatro estereoisómeros, en forma de aceite viscoso, químicamente inestable a temperatura ambiente y $\mathrm{pH}$ extremos. Por esta razón, las formas farmacéuticas utilizan una dispersión del principio activo en hidroximetilpropilcelulosa para su estabilización ${ }^{6}$, lo cual permite la formulación del medicamento en comprimidos. Esta forma farmacéutica es estable a temperatura ambiente y una alternativa muy conveniente a otros medicamentos uterotónicos como oxitocina y otras prostaglandinas que son formuladas como inyectables ${ }^{7}$.

\section{$\underline{\text { Propiedades farmacocinéticas }}$}

Luego de su administración, el misoprostol es rápidamente desesterificado a su ácido libre, el cual se cree es la forma activa del misoprostol.
El ácido misoprostólico tiene $85 \%$ de unión a proteínas plasmáticas y tiene una vida media de aproximadamente 30 minutos. Es excretado en la orina bajo la forma de diversos metabolitos inactivos. No se han descrito interacciones significativas con otros fármacos ${ }^{8}$.

El ácido misoprostólico es rápidamente absorbido desde distintos sitios de administración, las que incluyen oral, bucal, sublingual, rectal y vaginal, siendo esta última una vía de administración común en procedimientos obstétricos ya que la biodisponibilidad tras administración vaginal puede ser hasta tres veces más alta que la alcanzada por vía oral. De esta forma, la concentración máxima en plasma y el tiempo para alcanzar la concentración máxima dependen de la ruta de administración. Un resumen de estudios que comparan la farmacocinética por distintas rutas de administración se encuentra en la Tabla 1.

\section{Toxicidad}

Prácticamente no existen estudios disponibles en la literatura que evalúen la toxicidad del medicamento. No obstante, los estudios en animales de experimentación llevados a cabo mientras se desarrollaba el medicamento, demostraron que el medicamento no tiene una potencia importante como tóxico agudo ${ }^{14}$. Por ejemplo, la toxicidad oral aguda después de una dosis única permitió determinar un margen de seguridad de al menos 500 a 1.000 veces entre dosis letal en animales de experimentación y la dosis terapéutica en humanos. Asimismo, estudios de toxicidad crónica de 52 semanas con dosis de hasta $9.000 \mu \mathrm{g} / \mathrm{kg}$ en 
Tabla 1. Comparación de parámetros farmacocinéticos de misoprostol administrado por distintas vías

\begin{tabular}{|c|c|c|c|c|c|c|}
\hline $\begin{array}{l}\text { Vía de admi- } \\
\text { nistración }\end{array}$ & $\begin{array}{l}\text { Dosis } \\
(\mu g)\end{array}$ & $\begin{array}{c}\mathrm{n} \text { de } \\
\text { pacientes }\end{array}$ & $\begin{array}{l}\mathrm{Cmax}^{\mathrm{a}} \\
(\mathrm{pg} / \mathrm{ml})\end{array}$ & $\begin{array}{l}\left.\operatorname{Tmax}_{(\min }\right) \\
\end{array}$ & AUC (horas)c & Ref. \\
\hline Oral & 400 & 10 & $277 \pm 124$ & $34 \pm 17$ & $273,3 \pm 110,0 \quad(A \cup C 0-6)$ & 9 \\
\hline Oral & 400 & 10 & $287,6 \pm 144,3$ & $27,5 \pm 14,8$ & $402.8 \pm 151,6 \quad(A \cup C 0-6)$ & 10 \\
\hline Oral & 400 & 9 & $258,7(145-372)$ & $14,2(7,5-30)$ & $151,8(56,8-222,4) \quad(A \cup C 0-4)$ & 11 \\
\hline Oral & 800 & 8 & $484(350-2.030)$ & $30(22,5-45)$ & $380(223-1.470)(A \cup C 0-\infty)$ & 12 \\
\hline Oral & 400 & 10 & $264,8 \pm 170,7$ & $84,0 \pm 81,9$ & $475,2 \pm 312,9 \quad(A \cup C 0-4)$ & 13 \\
\hline Sublingual & 400 & 10 & $574,8 \pm 250,7$ & $26,0 \pm 11,5$ & $743,7 \pm 291,2 \quad($ AUC 0-6) & 10 \\
\hline Sublingual & 800 & 10 & $1.140(817-2.060)$ & $30(30-30)$ & $1.600(1.100-2.370)(A \cup C 0-\infty)$ & 12 \\
\hline Vaginal & 400 & 10 & $165 \pm 86$ & $80 \pm 27$ & $503,3 \pm 296,7 \quad(A \cup C 0-6)$ & 9 \\
\hline Vaginal & 400 & 10 & $125,2 \pm 53,8$ & $72,0 \pm 31,6$ & $433,7 \pm 182,6 \quad(A \cup C 0-6)$ & 10 \\
\hline Vaginal & 400 & 9 & $210,8(95-283)$ & $65,0(45-120)$ & $446,0(129,0-647,4) \quad(A \cup C$ 0-4) & 11 \\
\hline Vaginal & 400 & 10 & $445,9 \pm 428,7$ & $91,5 \pm 82,0$ & $925,5 \pm 567,5 \quad(A \cup C 0-4)$ & 13 \\
\hline Vaginal húmedod & 400 & 10 & $162,8 \pm 57,1$ & $75,0 \pm 31,6$ & $649,3 \pm 333,8 \quad(A \cup C 0-6)$ & 10 \\
\hline Vaginal húmedo & 400 & 10 & $427,1 \pm 235,5$ & $51,0 \pm 20,2$ & $1126,1 \pm 748,2 \quad($ AUC 0-4) & 13 \\
\hline Rectal & 400 & 9 & $86,8(59,0-200)$ & $71,7(45-120)$ & $188,9(46.1-442,6)(A \cup C 0-4)$ & 11 \\
\hline Rectal & 400 & 10 & $202,2 \pm 195,7$ & $19,5 \pm 14,2$ & $280,9 \pm 275,5 \quad(A \cup C 0-4)$ & 13 \\
\hline
\end{tabular}

Los datos se expresan según la publicación original, ya sea como media \pm desviación estándar o como media (rango).

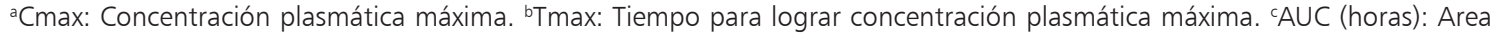
bajo la curva calculada para el intervalo de tiempo indicado. dvaginal húmedo: los comprimidos son humedecidos con gotas de solución salina antes de la administración. Ref.: referencia.

ratas demostraron sólo una hiperplasia gástrica reversible al discontinuar el medicamento. La mutagenicidad y la carcinogenicidad en ratas fueron también negativas. Notablemente, la fetotoxicidad y la teratogenicidad en ratas a dosis orales de hasta $10.000 \mu \mathrm{g} / \mathrm{kg}$ o en conejos en dosis de hasta $1.000 \mu \mathrm{g} / \mathrm{kg}$ fueron también negativos, aunque se observaron resorciones en un conejo y en una rata. En otro estudio, tampoco se encontró evidencia de embriotoxicidad a $20 \mathrm{mg} / \mathrm{kg}$ cuando el medicamento se administró a ratonas preñadas en el día de gestación $10^{15}$.

\section{Toxicidad sobre la gestación}

Aun cuando no se han descrito defectos del desarrollo en modelos animales, en humanos, la exposición in utero a misoprostol ha sido asociada con una serie de defectos que incluyen artrogriposis y reducción de extremidades, anormalidades cerebrales, gastrosquisis y síndrome de Moebius². Esta variedad de defectos fue reconocida por primera vez en Brasil durante la década de 1980-89 y hoy se reconoce una marcada asociación epidemiológica entre la exposición al medicamento en diversos países del mundo, sobre todo en aquellos donde la administración de misoprostol se realiza sin supervisión médica por ser el aborto un procedimiento no permitido.

La Tabla 2 resume estudios reporte de casos y caso-control aparecidos en la literatura científica desde 1998. La tabla sólo incluye casos en los que misoprostol se administró como agente único, excluyendo informes de efectos causados por la asociación de misoprostol a otros medicamentos como mifepristona o metotrexato.

De acuerdo a la tabla, los efectos causados por misoprostol se podrían resumir en tres tipos:

1. Defectos de extremidades. Deformidades superiores e inferiores, las que incluyen con frecuencia el pie varo-equino. En algunos casos, las anormalidades de extremidades están asociadas con artrogriposis, un conjunto de contracturas de articulaciones que llevan a atrofia muscular y a acumulación de tejido 
Tabla 2. Estudios reporte de casos y caso control que ligan la exposición in utero al misoprostol con la inducción de defectos del desarrollo

\section{Reporte}

Reporte de caso. Brasil. 42 niños de São Paulo, expuestos durante los primeros tres meses de gestación, y nacidos con defectos congénitos. 17 niños tenían pie equino y defectos de nervios craneanos. 10 niños tenían pie equino como parte de una artrogriposis. Los fenotipos más distintivos fueron artrogriposis de las piernas (cinco casos) y defectos de extremidades (nueve casos) con o sin síndrome de Möebius. La dosis declarada con mayor frecuencia fue de 800 $\mu \mathrm{g}$ (rango 200-16,000 $\mu \mathrm{g}$ ).

Reporte de caso. Sudáfrica. Niña nacida con un dígito extra en cada mano y con ausencia de la extremidad inferior derecha por debajo de la rodilla. El aborto se intentó en la semana 13 y el misoprostol se administró 1 comprimido vaginal por cuatro días consecutivos.

Estudio caso-control. Brasil. 96 niños con síndrome de Möebius fueron pareados con 96 niños con defectos del tubo neural para comparar la frecuencia de uso de misoprostol en ambos grupos. Entre las madres de los 96 niños con síndrome de Möebius, 47 (49\%) habían usado misoprostol en el primer trimestre de embarazo, en comparación con $3(3 \%)$ de las madres de los 96 niños con defectos del tubo neural (odds ratio, 29,7; 95 intervalo de confianza, $11,6$ a 76,0$)$.

Reporte de caso. Estados Unidos. Feto de un aborto electivo en la semana 17, previamente expuesto a $1.200 \mu \mathrm{g}$ de misoprostol durante la semana 7. El feto tenía manifestaciones variadas incluyendo omfaloceles, ausencia de falanges, amputación del pie izquierdo y síndrome de banda amniótica.

Reporte de caso. Brasil. Niño con síndrome de Möebius, falla respiratoria y posible daño al tallo cerebral. La exposición al misoprostol ocurrió en la semana de gestación 8.

Estudio caso-control. Brasil. Estudio prospectivo, observacional, de cohorte, con 86 casos expuestos a misoprostol y 86 casos pareados y no expuestos. No hubo diferencias significativas en la tasa de defectos mayores o menores entre los niños expuestos comparados con los no-expuestos (2/67 vs 2/81, defectos mayores; $7 / 67$ vs 3/81, anomalías menores). Las dosis totales administradas variaron entre un comprimido $(200 \mu \mathrm{g})$ a 98 comprimidos $(19,600 \mu \mathrm{g})$, en una administración única de 1 a 4 comprimidos vía oral u oral y vaginal, 5 a 8 semanas después de la última menstruación.

Estudio caso-control. Brasil. Los casos correspondieron a 26 recién nacidos que pesaron menos de $1.500 \mathrm{~g}$ con anomalías congénitas de distintos tipos mientras que el grupo de comparación incluyó a 276 niños también de menos de $1.500 \mathrm{~g}$ pero sin defectos. El odds ratio multivariado de tener un hijo con anomalías congénitas fue de 2,4 (intervalo de confianza del $95 \% ; 1,0-6,2$ ) en madres que señalaron haber utilizado misoprostol en comparación con las madres que utilizaron el medicamento.

Reporte de caso. Brasil. Quince casos of artrogriposis. En todos los casos se observó también retraso en el crecimiento, huesos subdesarrollados, pies pequeños y varos, rigidez de articulaciones, falta de movilidad de las piernas secundaria a alteraciones neurológicas, hipoplasia simétrica bilateral o atrofia de la musculatura de extremidades y reflejos tendinianos ausentes. En cinco casos se verificó deformidades en las extremidades superiores y en un caso se observó disrupción de la médula espinal que originó pérdida sensorial segmentada y vejiga neurogénica. Misoprostol fue administrado durante las semanas 8 a la 12, en dosis entre 400 y $4.800 \mu \mathrm{g}$ por vía oral o vaginal.

Estudio caso-control. Brasil. 4.673 recién nacidos consecutivos con malformaciones de etiología desconocida, pertenecientes al registro del Estudio Colaborativo Latino Americano de Malformaciones Congénitas (ECLAM). No hubo diferencias entre exposición a misoprostol en recién nacidos malformados (34/4.673) y no malformados (23/4.980) aunque se encontró un exceso de defectos de extremidades, hidrocefalos y artrogriposis. La dosis y la vía de administración fueron pobremente especificadas. En la mayoría de los casos, la exposición a misoprostol ocurrió durante el primer trimestre.

Estudio caso-control. Brasil. Comparación de la frecuencia de uso de misoprostol entre madres de niños diagnosticados con defectos inducidos por disrupción vascular y madres de niños con otros tipos de defectos. Se estudió un total de 93 casos y 279 controles en 8 centros asistenciales. La exposición prenatal fue identificada en 32 casos de defectos de disrupción vascular (34,4\%) en comparación con sólo $12(4,3 \%)$ en el grupo control $(p<0,0000001)$. La mayoría de las madres utilizaron 4 comprimidos (dosis total $800 \mu \mathrm{g}$ ) ya sea oral o vaginal entre las semanas 5 a 8 después del último período menstrual.

Reporte de caso. Brasil. Estudio de 23 niños con síndrome de Möebius, de los cuales 3 tenían autismo concomitante y 4 presentaban alguna manifestación del espectro autistístico. La dosis y la ruta no se informan aunque se señala que la exposición a misoprostol ocurrió durante el primer trimestre. 
Tabla 2. Estudios reporte de casos y caso control que ligan la exposición in utero al misoprostol con la inducción de defectos del desarrollo (continuación)

\section{Reporte}

Reporte de caso. Venezuela. Niño nacido con síndrome de Möebius. La madre trató de abortar dos veces durante el primer trimestre de embarazo, utilizando, en ambas oportunidades $600 \mu \mathrm{g}$ vaginal y $900 \mu \mathrm{g}$ oral.

Reporte de una asociación observada entre el uso de misoprostol durante el primer trimestre y malformaciones congénitas o muerte fetal en el Estudio Brasilero de Diabetes Gestacional, una cohorte de mujeres en atención prenatal en hospitals públicos de 6 ciudades. Las mujeres expuestas tenían un mayor riesgo de tener un hijo con malformaciones $(R R=2,39)$, que incluían sindactilia, pie equino, meningomielocele, microcefalia, y defectos inguinales. Muerte fetal también fue significativamente mayor en el grupo de madres expuestas $(R R=2,63)$

Reporte de caso. Pakistan. Niña que nace con malformaciones craneofaciales con protrusión de la duramadre, microcefalia y pie equino derecho. La madre intentó aborto con $400 \mu \mathrm{g}$ of misoprostol oral por dos días a las 8 semanas de gestación.

Meta análisis de cuatro estudios caso-control (Brasil et al, 2000; Oriolli and Castilla, 2000; Pastuszack et al, Vargas et al, 2000) con un total de 4.899 casos de anomalías congénitas y 5.742 controles. Se encontró un incremento de riesgo de anomalías congénitas para cualquier defecto $(\mathrm{OR}=3,56 ; 95 \% \mathrm{Cl}: 0,98-12,98)$, síndrome de Möebius $(\mathrm{OR}=25,31 ; 95 \% \mathrm{Cl}: 11,11-57,66)$ y defectos de extremidades (OR $=11,86 ; 95 \% \mathrm{Cl}: 4,86-28,90)$.

Reporte de caso. Chile. Niño nacido con síndrome de Möebius y artrogriposis. La madre intentó un aborto a las 8 semanas de gestación con tres comprimidos $(600 \mu \mathrm{g})$ administrados intravaginalmente.

Reporte de caso. Brasil. Niño de un año con pliegues epicánticos bilateral, aplasia esternocostal del músculo pectoral con pezón derecho hipoplástico y extremidades superiores asimétricas. Un estudio angiotomográfico reveló la presencia de la arteria subclavia aberrante. Un estudio oftalmológico evidenció una tortuosidad bilateral intense de los vaso de la retina con estrechamiento arterial y degeneración del epitelio retinal. La madre utilizó misoprostol alrededor de la semana 6 ó 7 de gestación, $400 \mu \mathrm{g}$ oral y $400 \mu \mathrm{g}$ vaginal.

Segunda evaluación de mujeres reclutadas en el Estudio Brasilero de Diabetes Gestacional. 120 mujeres (17\%) señalaron haber utilizado misoprostol durante el primer trimestre. Se encontró una asociación positiva entre el uso de misoprostol y las anomalías congénitas ( $\mathrm{OR}=2,64 ; 95 \% \mathrm{Cl}: 1,03-6,75)$.

Reporte de caso. Colombia. Siete niños con dismorfología al nacer incluyendo síndrome de Möebius (3), síndrome hipoglosia-hipodactilia (1), ciclopía (1), gastrosquisis (1), microcefalia (1), secuencia de Klippel Fiel (1) e hidroanencefalia (1). La exposición a misoprostol ocurrió durante las semanas de gestación 4 a 14 y las dosis fluctuaron entre 800 y $1,000 \mu \mathrm{g}$

Reporte de caso. Colombia. Recién nacido con aglosia y adactilia. La madre utilizó una combinación de misoprostol $400 \mu \mathrm{g}$ oral y $200 \mu \mathrm{g}$ intravaginal en la semana 10 de gestación.

Reporte de caso. Brasil. Niña de siete meses con hipotonía, alteraciones faciales y de extremidades, ano imperforado, hipoplasia pélvica, atresia esofágica y defecto atrio-septal. La madre utilizó una dosis de misoprostol de $600 \mu \mathrm{g}$ (tres comprimidos) durante el segundo mes de embarazo.

Reporte de caso. Brasil. Niña de diez meses con síndrome de Moebius y holoprosencefalia. La madre utilizó misoprostol pero no especifica dosis ni semana de gestación.

Ref.: referencia.

fibroso alrededor de las articulaciones.

2. Defectos del sistema nervioso. Principalmente síndrome de Moebius (también conocido como secuencia de Moebius) el cual es una condición poco frecuente que se caracteriza por parálisis congénita de los nervios craneanos sexto y séptimo, con o sin parálisis de otros nervios craneanos. Se manifiesta como parálisis de la cara, estrabismo, hipoplasia de la lengua y se acompaña frecuentemente por defectos de extremidades. Recientemente se ha propuesto también que misoprostol puede inducir manifestaciones del espectro autístico asociado con malformaciones y disfunciones tales como el síndrome de Moebius $^{37,38}$.

3. Otros. Anormalidades viscerales y esqueléticas, defectos del tubo neural y labio y 
paladar fisurado.

Los primeros estudios epidemiológicos, que analizaban datos de la década de 1990-99, mostraban sólo una débil asociación entre exposición a misoprostol y la inducción de defectos congénitos. Por ejemplo, en el estudio de Schuler y $\mathrm{col}^{21}$, los autores estudiaron 86 mujeres expuestas a misoprostol, las que fueron pareadas con un número similar de mujeres no expuestas. La falta de diferencias significativas en las tasas de defectos entre ambos grupos llevó a los autores a concluir que el uso de misoprostol durante el embarazo podría aumentar la incidencia de anomalías congénitas pero que la magnitud de este incremento era baja. El aumento en el número de casos al trascurrir el tiempo ha permitido mejorar el poder estadístico para establecer la magnitud del riesgo de inducción de defectos congénitos por misoprostol.

De esta forma, el estudio más extenso disponible en la literatura es una revisión sistemática de da Silva dal Pizzol y col $^{29}$. Los autores estimaron un riesgo de anormalidades congénitas y otros efectos adversos en niños expuestos a misoprostol durante su vida fetal, utilizando cuatro estudios caso-control ${ }^{4,18,21,23}$ los que totalizan 4.899 casos de anormalidades congénitas y 5.742 controles. Los autores encontraron riesgos aumentados para defectos congénitos relacionados con misoprostol para cualquier defecto $(\mathrm{OR}=3,56$; $95 \%$ CI: 0,98 $12,98)$, síndrome de Moebius ( $\mathrm{OR}=25,31 ; 95 \%$ CI: $11,11-57,66)$ y defectos de extremidades $(\mathrm{OR}=$ 11,86; 95\% CI: 4,86-28,90). Estos altos odds ratios indudablemente asocian la exposición fetal al misoprostol con la inducción de defectos congénitos.

Aun cuando, la mayoría de los estudios presentados en la Tabla 2 señalan que existe gran sesgo de memoria en la capacidad de la madre para recordar la dosis y el día de gestación en que tomó misoprostol, en la mayoría de los casos se informa que la administración del medicamento fue durante el primer trimestre, más específicamente alrededor o después de las 8 semanas, usualmente en una combinación de administración oral y vaginal y en dosis que fluctúan entre 400 y $1.800 \mu \mathrm{g}$. El período de sensibilidad del desarrollo de extremidades es entre las 5 y las 8 semanas de gestación, mientras que para el sistema nervioso central el período crítico abarca todo el primer semestre. En este caso, la formación de ganglios craneanos, cuya anomalía sería la base para el síndrome de Moebius, sería alterada por efecto del medicamento. De esta forma, las exposiciones humanas al misoprostol informadas y revisadas por las publicaciones de la Tabla 2 coinciden con los períodos de sensibilidad fetal y refuerzan la plausibilidad biológica para la inducción de defectos del desarrollo por misoprostol.

La falta de observaciones en animales de experimentación es llamativa y puede deberse simplemente a que los modelos animales utilizados hasta el momento no responden al medicamento del mismo modo que los humanos. Shepard ${ }^{39}$ ha señalado que la teratogenicidad en animales de experimentación es importante pero no esencial para evidenciar el efecto de las sustancias químicas en humanos, reconociendo así que en muchas ocasiones puede que no haya un modelo animal adecuado para estudiar la toxicidad sobre la gestación. En este contexto, el hecho de que misoprostol no sea teratogénico para los animales no es un argumento para negar sus efectos sobre humanos.

El mecanismo de toxicidad del misoprostol durante la gestación no ha sido dilucidado todavía. Debido a la similitud entre los defectos observados después de insultos por la hipoxia y aquellos observados en niños expuestos al misoprostol, una propuesta común es que el medicamento causa una disrupción del desarrollo en una etapa altamente dependiente de oxígeno. Esta disrupción puede ser causada por atrapamiento de la sangre fetal en la placenta por las contracciones uterinas ${ }^{40}$ o por un efecto vasoconstrictor sobre las arterias uterinas ${ }^{41}$. Yip y col $^{42}$ utilizaron ecografía Doppler para medir efectos vasoactivos en mujeres que tomaron 200 $\mu \mathrm{g}$ de misoprostol y confirmaron un aumento en la resistencia de la arteria uterina, lo cual implica una reducción en el flujo sanguíneo y concuerda con el mecanismo propuesto.

\section{Conclusión}

El misoprostol es considerado, en países donde el aborto es legal, como un medicamento seguro de utilizar con este fin si es que se utiliza bajo prescripción médica y en establecimientos clínicos. Si el procedimiento falla, un segundo intento con misoprostol o un aborto quirúrgico debe ser ofrecido a la paciente ${ }^{1}$ ya que, si el embarazo continúa la exposición in utero al medicamento conlleva un riesgo considerable de toxicidad sobre el desarrollo embrionario. De esta forma, se 
concluye que misoprostol es un teratógeno. Sin embargo, limitaciones en el reporte de casos (por ejemplo, se desconoce cuántos abortos se realizan, cuántos fallan y cuántos utilizan misoprostol) no permiten establecer la real dimensión de su potencia teratogénica.

\section{Referencias}

1. Faúndes A, Fiala C, Tang OS, Velasco A. Misoprostol for the termination of pregnancy up to 12 completed weeks of pregnancy. Intern J Gynecol Obstet 2007; 99: S172-7.

2. Schardein JL, Macina OT. Human Developmental Toxicants. Aspects of Toxicology and Chemistry. Boca Ratón, Estados Unidos: CRC Press; 2007.

3. Schuler L, Pastuszak A, Sanseverino MTV, Orioli IM, Brunoni D, Ashton-Prolla P, et al. Pregnancy outcome after exposure to misoprostol in Brazil: A prospective, controlled study. Reprod Toxicol 1999; 13: 147-51.

4. Orioli IC, Castilla E. Epidemiological assessment of misoprostol teratogenicity. Brit J Obstet Gynaecol 2000; 107: 519-23.

5. Philip N, Shannon C, Winikoff B. Misoprostol and teratogenicity: reviewing the evidence. New York, Estados Unidos: The Population Council; 2003.

6. Collins PW, Pappo R, Dajani EZ. Chemistry and synthetic development of misoprostol. Dig Dis Sci 1985; 30 (Suppl): 114S-7S.

7. Elati A, Weeks A. The use of misoprostol in obstetrics and gynaecology. BJOG 2009; 116 (Suppl 1): 61-9.

8. Davies NM, Longstreth J, Jamali F. Misoprostol therapeutics revisited. Pharmacotherapy 2001; 21: 60-73.

9. Zieman M, Fong S, Benowitz N, Bankster D, Darney PD. Absorption kinetics of misoprostol with oral or vaginal administration. Obstet Gynecol 1997; 90: 88-92.

10. Tang OS, Schweer H, Seyberth HW, Lee SW, Ho PC. Pharmacokinetics of different routes of administration of misoprostol. Hum Reprod 2002; 17: 332-6.

11. Khan R-U, El-Refaey H, Sharma S, Sooranna D, Stafford M. Oral, rectal, and vaginal pharmacokinetics of misoprostol. Obstet Gynecol 2004; 103: 866-70.

12. Schaff EA, DiCenzo R, Fielding SL. Comparison of misoprostol plasma concentrations following buccal and sublingual administration. Contraception 2005; 71: 22-5.

13. Meckstroth KR, Whitaker AK, Bertisch S, Goldberg AB, Darney PD. Misoprostol administered by epithelial routes. Drug absorption and uterine response. Obstet Gynecol 2006; 108: 582-90.

14. Kotsonis FN, Dodd DC, Regnier B, Kohn FE. Preclinical toxicology profile of misoprostol. Dig Dis Sci 1985: 30
(Suppl): 142S-6S.

15. Paumgarten FJR, Souza CAM, Carvalho RR, Chahoud I. Embryotoxic effects of misoprostol in the mouse. Braz J Med Biol Res 1995; 28: 355-61 (resumen).

16. González CH, Marques-Dias MJ, Kim CA, Sugayama SMM, da Paz JA, Huson SM, et al. Congenital abnormalities in brazilian children associated with misoprostol misuse in first trimester of pregnancy. Lancet 1998; 351: 1624-7.

17. Hofmeyr GJ, Milos O, Nikodem NC, de Jager M. Limb reduction anomaly after failed misoprostol abortion. SAMJ 1998; 88: 566-7.

18. Pastuszak AL, Schuler L, Speck-Martins CE, Coelho KEFA, Cordello SM, Vargas F, et al. Use of misoprostol during pregnancy and Mobius' Syndrome in infants. N Engl J Med 1998; 338: 1881-5.

19. Genest DR, Di Salvo D, Rosenblatt MJ, Homes LB. Terminal transverse limb defects with tethering and omphalocele in a 17 week fetus following first trimester misoprostol exposure. Clin Dys 1999; 8: 53-8.

20. Nunes ML, Friedrich MAG, Loch LF. Association of misoprostol, Möebius syndrome and congenital central alveolar hypoventilation. Arq Neuropsiquiatr 1999; 57: 88-91.

21. Schuler L, Pastuszak A, Sanseverino MTV, Orioli IM, Brunoni D, Ashton-Prolla P, et al. Pregnancy outcome after exposure to misoprostol in Brazil: A prospective, controlled study. Reprod Toxicol 1999; 13: 147-51.

22. Brasil R, Coelho HL, D’Avanzo B, La Vecchia C. Misoprostol and congenital anomalies. Pharmacoepidemiol Drug Saf 2000; 9: 401-3.

23. Coelho K-E, Sarmento M, Veiga CM, Speck-Martins CE, Safatle HPN, Castro C, et al. Misoprostol Embryotoxicity: Clinical Evaluation of Fifteen Patients With Arthrogryposis. Am J Med Gen 2000; 95: 297-301.

24. Vargas FR, Schuler-Faccini L, Brunoni D, Kim C, Meloni VFA, Suguyama, SMM, et al. Prenatal exposure to misoprostol and vascular disruption defects: A case-control study. Am J Med Genet 2000; 95: 302-6.

25. Bandim JM, Ventura LO, Miller TM, Almeida HC, Santos Costa AE. Autism and Möbius sequence. An exploratory study of children in northeastern Brazil. Arq Neuropsiquiatr 2003; 61 (2-A): 181-5.

26. Sánchez O, Guerra D. Síndrome de Möebius: fetopatía por misoprostol. Reporte de un paciente. Invest Clin 2003; 44: 147-53.

27. da Silva Dal Pizzol T, Tierling VL, Schüler-Faccin L, Sanseverino MTV, Mengue SS. Reproductive results associated with misoprostol and other substances utilized for interruption of pregnancy. Eur J Clin Pharmacol 2005; 61: 71-2. 
28. Ara Qazi R. Congenital anomalies associated with failed misoprostol termination of pregnancy. JLUMHS 2006; 5: 122-4.

29. da Silva dal Pizzol T, Pozzobon Knop F, Mengue SS. Prenatal exposure to misoprostol and congenital anomalies: Systematic review and meta-analysis. Reprod Toxicol 2006; 22: 666-71.

30. Osorio A, Rodríguez JG, Pizarro O, Paredes A, Aguilera S. Embriopatías asociadas al uso de misoprostol. Rev Chil Obstet Ginecol 351; 72: 346-51.

31. Rosa RF, Travi GM, Valiatti F, Zen PL, Pinto LL, Kiss A, et al. Poland syndrome associated with an aberrant subclavian artery and vascular abnormalities of the retina in a child exposed to misoprostol during pregnancy. Birth Defects Res (Part A) 2007; 79: 507-11.

32. da Silva dal Pizzol T, Sanseverino MTV, Mengue SS. Exposure to misoprostol and hormones during pregnancy and risk of congenital anomalies. Cad Saúde Pública 2008; 24:1447-53.

33. Isaza C, Saldarriaga W, Pachajoa H. Uso inadecuado de misoprostol. ¿Un problema de salud pública? Colomb Med 2008; 39 (Supl 2): 61-5.

34. Ramírez-Cheyne J, Pachajoa H, Isaza C, Saldarriaga W. Síndrome de aglosia-adactilia y exposición prenatal a misoprostol ¿relación causal o casual? Reporte de un caso. Rev Col Obstet Ginecol 2008; 59: 248-52.
35. Bernardi P, Graziadio P, Machado-Rosa R, Nunes Pfeil J, Gazzola Zen P, Paskulin G. Fibular dimelia and mirror polydactyly of the foot in a girl presenting additional features of the VACTERL association. Sao Paulo Med J 2010; 128: 99-101.

36. Pirmez R. Freitas ME, Gasparetto E, Araujo A. Moebius Syndrome and Holoprosencephaly Following Exposure to Misoprostol. Pediatr Neurol 2010; 43: 371-3.

37. Miller MT, Strömland K, Ventura L, Johansson M, Ban$\operatorname{dim}$ JM, Gillber C. Autism associated with conditions characterized by developmental errors in early embryogenesis: a mini review. Int. J. Devl Neuroscience 2005; 23: 201-19.

38. Miller MT, Strömland K, Ventura L. Congenital Aberrant tearing: a re-look. Trans Am Ophthalmol Soc 2008; 106 : 100-16.

39. Shepard TH. Proof of human teratogenicity. Teratology 1994; 50: 97-8.

40. Los FJ, Brandenburg H, Niermeijer MF. Vascular disruptive syndromes after exposure to misoprostol or chorionic villus sampling. The Lancet 1996; 353: 843-4.

41. Webster WS, Abela D. The effect of hypoxia in development. Birth Def Res(Part C) 2007; 81: 215-28.

42. Yip S-K, Tse A, Haines CJ, Chung T. Misoprostol's effect on uterine arterial blood flow and fetal heart rate in early pregnancy. Obstet Gynecol 2000; 95: 232-5. 